\section{AIDS em crianças: a influência dos diferenciais socioeconômicos no Município de Fortaleza, Ceará, Brasil}

\author{
AIDS in children: the influence of socioeconomic \\ differences in Fortaleza, Ceará State, Brazil
}

\author{
SIDA en niños: la influencia de las diferencias \\ socioeconómicas en la ciudad de Fortaleza, \\ Ceará, Brasil
}

Abstract

\footnotetext{
1 Universidade Federal do Ceará, Fortaleza, Brasil.

2 Universidade Federal de Pernambuco, Recife, Brasil.

Correspondência

E. M. Lopes

Universidade Federal

do Ceará.

Av. Juscelino Kubitschek 3700 Bloco C, sala 404, Fortaleza,

CE 60861-630, Brasil.

emelinepet@yahoo.com.br
}

This study aimed to analyze the relationship between pediatric AIDS cases and neighborhood socioeconomic characteristics in Fortaleza, Ceará State, Brazil. This was an ecological and descriptive study with socioeconomic data from the 2010 Population Census and AIDS data in children for 2001-2011 from the Information System on Notifiable Diseases. Pearson's correlation was performed and the Moran index was used to verify spatial autocorrelation. A multinomial model was used to identify clusters. Of the total sample, $57.8 \%$ were male. In $18.8 \%$ of the children, the diagnosis was made after two years of age. There was a positive spatial correlation between AIDS cases in children and income $(I=0.6 ; p=0.001)$ and the proportion of poor ( $I=0.5 ; p=0.001)$. Neighborhoods with these characteristics are mainly located in the Northwest and Southwest areas of the city. Spatial correlation between pediatric AIDS cases and socioeconomic indicators highlights the need for interventions aimed at improving health and living conditions.

Acquired Immunodeficiency Syndrome; Child; Socioeconomic Factors
Emeline Moura Lopes 1

Nathália Lima Pedrosa 1

Eliane Rolim de Holanda 2

Rosa Lívia Freitas Almeida 1

Lígia Regina Franco Sansigolo Kerr 1

Marli Teresinha Gimeniz Galvão ${ }^{1}$

\section{Resumo}

Objetivou-se analisar a relação entre casos de AIDS em crianças e características socioeconômicas de bairros de Fortaleza, Ceará, Brasil. Estudo ecológico descritivo, cujos dados socioeconômicos foram obtidos do Censo Demográfico de 2010 e os de AIDS em crianças referentes ao período de 2001 a 2011 no Sistema de Notificação de Agravos. Realizou-se análise de correlação de Pearson e utilizou-se o índice de Moran para verificar a autocorrelação espacial. Adotou-se o modelo multinomial para identificar clusters. Verificou-se 57,8\% das crianças do sexo masculino. Em 18,8\% das crianças o diagnóstico ocorreu após os dois anos de idade. Observou-se correlação espacial positiva dos casos de AIDS em crianças em relação à renda $(I=0,6 ; p=0,001)$ e à proporção de pobres $(I=0,5 ; p=0,001)$. Os bairros com essa caracterização estão localizados principalmente ao noroeste e sudoeste da cidade. A correlação espacial dos casos de AIDS em crianças com os indicadores socioeconômicos evidencia a necessidade de intervenções voltadas à saúde e melhoria das condições de vida.

Síndrome de Imunodeficiência Adquirida; Criança; Fatores Socioeconômicos 


\section{Introdução}

As crianças nascidas de mães soropositivas ao HIV constituem um grupo de vulnerabilidade para a infecção pelo HIV, com nítidos aumentos da incidência de recém-nascidos infectados 1 . Diversos fatores conduzem esse grupo a essa vulnerabilidade, tais como a dependência do cuidado para diagnosticar precocemente o vírus durante a gestação e após o nascimento, fornecimento do tratamento antirretroviral e acompanhamento nos serviços de saúde; o sistema educacional (creches/escolares); e as condições de pauperização e baixa escolaridade da criança e do familiar/cuidador 2 .

No Brasil, percebe-se a concentração de casos de AIDS em regiões de maior pobreza, o que evidencia a relação entre AIDS e as condições socioeconômicas $3,4,5$. No contexto da transmissão vertical, a maior ocorrência também é em regiões menos favorecidas 4 .

Nos países subdesenvolvidos, onde está concentrada a maior parte dos casos da doença, a infecção de jovens e crianças é alvo de preocupação governamental ${ }^{6}$. Tal aspecto reforça a necessidade de incorporação de indicadores socioeconômicos espacializados no território nos estudos de saúde da população, haja vista que constituem importantes ferramentas para subsidiar políticas e práticas de promoção da saúde que possibilitem a identificação de áreas de maior vulnerabilidade à infecção pelo HIV, com vistas ao direcionamento de intervenções mais efetivas.

A importância da incorporação de indicadores socioeconômicos está na possibilidade de discutir a ocorrência de doenças em diferentes grupos populacionais, proporcionando uma maior compreensão do espaço social e dos agravos a eles associados ?

Para isso a análise espacial tem se mostrado como uma importante ferramenta para a melhor compreensão da distribuição de doenças no território e sua relação com fatores ambientais e socioeconômicos 8, e os estudos ecológicos são essenciais tanto ao diagnóstico das tendências da epidemia quanto à proposição de estratégias preventivas.

Considerando que condições socioeconômicas desfavoráveis conferem maior vulnerabilidade à infecção pelo HIV/AIDS e que a identificação de áreas vulneráveis fornece subsídio às intervenções públicas em saúde, o presente estudo teve como objetivo analisar a distribuição espacial dos casos de AIDS em crianças e sua relação com indicadores socioeconômicos, no Município de Fortaleza, Estado do Ceará, Brasil.

\section{Método}

Estudo epidemiológico com desenho ecológico transversal, tendo como unidade de análise os 119 bairros oficiais do Município de Fortaleza compatibilizados com a malha censitária do Instituto Brasileiro de Geografia e Estatística (IBGE. Censo Demográfico 2010. http:// downloads.ibge. gov.br/downloads_geociencias.htm, acessado em 10/Out/2013).

Fortaleza é capital do Estado do Ceará, localizada no Nordeste do Brasil. Administrativamente está dividida em sete secretarias, sendo seis Secretarias Executivas Regionais (SER I a VI) e uma Regional Centro, que de forma articulada definem prioridades e metas específicas conforme as características epidemiológicas e sanitárias de cada área. As SER têm características sociais e econômicas similares, tendo entre 15 e 29 bairros. Dentre essas, a SER I possui o menor número de bairros e a maior densidade demográfica (14.338,53 habitantes/ $\mathrm{km}^{2}$ ), e a SER VI apresenta o maior número de bairros e a menor densidade (4.010,67 habitantes $\left./ \mathrm{km}^{2}\right)$, possuindo área que abrange $40,25 \%$ do total da cidade. Fortaleza conta com nove instituições de referência para o acompanhamento e tratamento de pessoas vivendo com HIV/AIDS, sendo que um serviço é exclusivo para crianças e adolescentes 9 .

O trabalho foi desenvolvido utilizando-se dados do Censo Demográfico de 2010, realizado pelo IBGE, e dados do Sistema Nacional de Agravos de Notificação (SINAN) obtidos junto à Secretaria Estadual de Saúde (SESA), referentes aos casos de AIDS em crianças entre 2001 e 2011.

Adotaram-se como critérios de inclusão dos casos de AIDS em crianças: notificações ocorridas entre 2001 e 2011; idade igual ou inferior a 12 anos; residentes no Município de Fortaleza. Ao todo foram contabilizados 90 casos de AIDS. $\mathrm{Na}$ fase de análise espacial, quatro casos foram excluídos por não constar o endereço na ficha de notificação.

Inicialmente, foram selecionadas as variáveis epidemiológicas sexo, raça da criança, escolaridade da mãe, idade da criança no período do diagnóstico, ano de diagnóstico, ano de notificação e bairro de residência. Contudo, verificando-se a frequência simples de preenchimento, as variáveis raça e escolaridade da mãe tiveram registros em $62 \%$ e $36,8 \%$ das fichas, respectivamente, sendo excluídas para análise por terem suas completudes consideradas como ruins e muito ruins 10 .

Como indicadores socioeconômicos foram elencados: proporção de pessoas responsáveis pelo domicílio, proporção de moradias adequadas, proporção de pessoas na faixa da pobreza 
(com renda de até meio salário mínimo), número de salários mínimos e proporção de pessoas analfabetas (número de pessoas acima de 15 anos que não sabem ler e escrever em seu idioma de origem), proporção de domicílios com banheiro de uso exclusivo ou sanitário, e proporção de domicílios com sete ou mais moradores. Esses indicadores foram construídos com base no agrupamento dos setores censitários fornecidos pelo IBGE e compatibilizados com a malha de bairros do Município de Fortaleza.

Realizou-se análise de correlação de Pearson entre a taxa de AIDS em crianças e os indicadores socioeconômicos. Consideraram-se eleitos para o modelo inicial aqueles fatores cuja correlação linear com o desfecho fosse estatisticamente significante no nível de $20 \%$ e simultaneamente não apresentassem correlação maior que $\geq 0,8$ entre os fatores 11. Posteriormente, foi empreendida regressão linear múltipla, com seleção de variáveis do tipo backward para identificar quais indicadores, segundo critérios de análise residual e coeficiente de determinação, melhor explicam o desfecho. Foram consideradas significantes as que apresentaram $\mathrm{p}<0,05$.

As análises descritivas apresentadas foram desenvolvidas no software SPSS (SPSS Inc., Chicago, Estados Unidos). A taxa de AIDS em crianças foi calculada para cada bairro, usando-se a razão entre o número de casos de AIDS notificados em menores de 12 anos e a respectiva população na mesma faixa etária, multiplicado por $100 \mathrm{mil}$.

Para verificar a existência de um padrão espacialmente condicionado da ocorrência de AIDS em criança utilizaram-se estatísticas de autocorrelação espacial. A presença de autocorrelação espacial foi mensurada pelo índice global de Moran (I), com o emprego da matriz de vizinhança da distância de centroide a centroide. Para testar a autocorrelação, a hipótese nula foi a de não correlação espacial, com média e variância conhecidas. A presença de autocorrelação espacial global implica que áreas próximas apresentam taxas mais semelhantes do que seria esperado caso sua distribuição fosse aleatória, indicando a ocorrência de dependência espacial para o evento sob estudo.

O índice de Moran (I) é um coeficiente de autocorrelação ponderado, cujos valores tendem a se situar entre -1 e +1 . Valores próximos a +1 indicam que os dados possuem autocorrelação espacial positiva. Valores próximos a -1 indicam que os valores não semelhantes tendem a se juntar, ou seja, um padrão de autocorrelação negativa. Valores próximos a zero indicam ausência de correlação espacial, isto é, um processo aleatório no espaço.
Foi realizada análise puramente espacial, com o uso do software SaTScan v. 8.0 (http:\। www.satscan.org, Estados Unidos). Adotou-se o modelo multinomial para identificar aglomerados de casos de AIDS em associação com as covariáveis proporção de analfabetos, proporção de pessoas na faixa de pobreza e renda média. A significância estatística foi testada por Monte Carlo para até 999 interações $\mathrm{p}<0,05$.

O estudo foi aprovado pelo Comitê de Ética em Pesquisa da Universidade Federal do Ceará, conforme protocolo no 328.835 .

\section{Resultados}

Entre as variáveis epidemiológicas selecionadas para análise, os campos sexo, idade no período do diagnóstico, ano de diagnóstico e ano de notificação não tiveram missing. Considere-se que todos esses campos são de preenchimento obrigatório no sistema, sem os quais o registro ficaria impossibilitado de ser concluído. O campo bairro de residência foi preenchido em 95,5\% dos registros, sendo considerada uma completude excelente para utilização dos dados para análise 11 .

Dos 90 casos de AIDS entre crianças, 57,7\% eram do sexo masculino. Quanto à idade no ano de diagnóstico, 18,8\% dos casos notificados correspondiam a crianças com dois anos de idade. Quanto ao ano de diagnóstico, foram identificados casos em todos os anos do intervalo, principalmente em 2011, correspondendo a 12,2\% dos registros de diagnóstico, e 2003 e 2008, com $11,1 \%$ dos casos em cada ano. Quanto às notificações, no ano de 2010 foi computada a maior parcela de casos, o equivalente a $17,7 \%$ do total notificado no período (Figura 1).

Foi avaliada a categoria de exposição dos 90 casos utilizados nessa fase, sendo verificado que $78(86,7 \%)$ deles tiveram a transmissão vertical como fonte de infecção. Em 2005, houve um caso $(1,1 \%)$ relacionado a transfusões. A categoria de exposição ao HIV foi ignorada ou não foi preenchida em $11(12,2 \%)$ registros.

Verificou-se a ocorrência de casos em 50 bairros de Fortaleza (42\%), localizados em sua maioria (80\%) nas SER I e III. As maiores taxas de AIDS em criança foram observadas nas SER I e III, 25,9 e 28,7 casos por 100 mil crianças, respectivamente. Na SER V, houve a maior proporção de pessoas na faixa de pobreza $(6,5 \%)$ e a maior proporção de analfabetos $(8,4 \%)$ (Tabela 1$)$.

Observou-se elevada correlação espacial positiva dos indicadores socioeconômicos renda média $(\mathrm{I}=0,6 ; \mathrm{p}=0,001)$ e proporção de pobres ( $\mathrm{I}=0,48 ; \mathrm{p}=0,001$ ), indicando uma distribuição de renda desigual (Tabela 2). Verificou-se corre- 


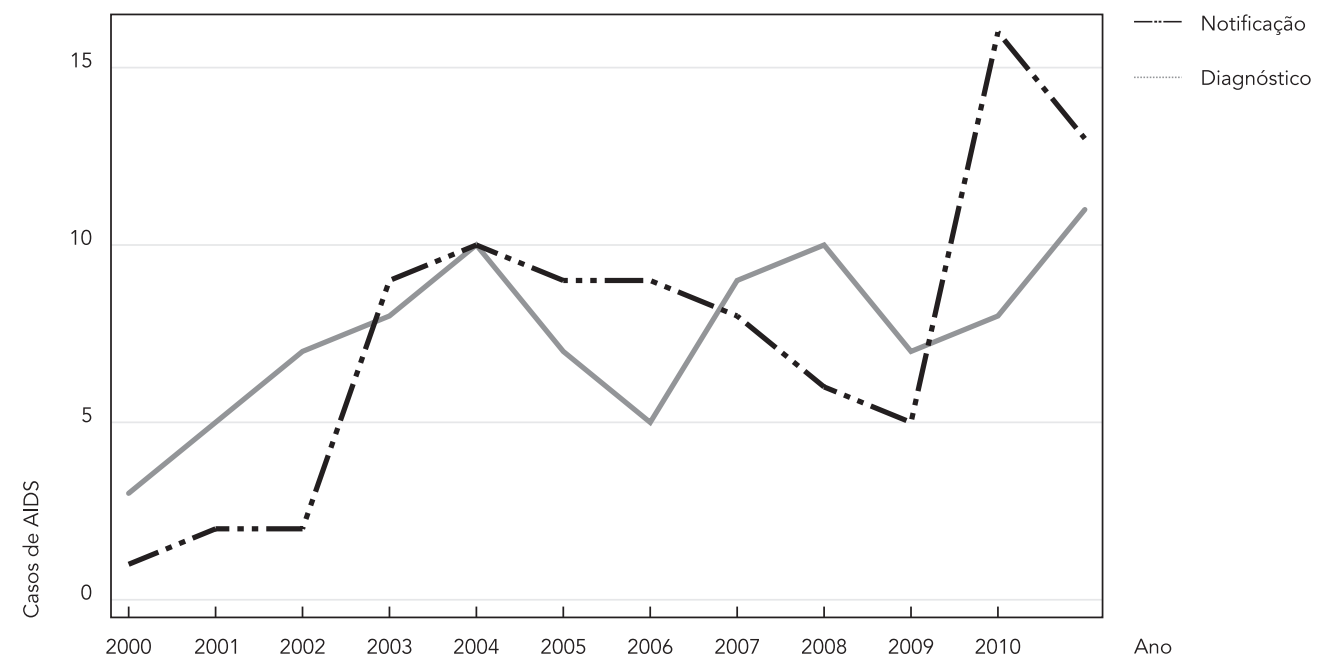

Tabela 1

Distribuição dos bairros, casos de AIDS em crianças e indicadores socioeconômicos de acordo com as Secretarias Executivas Regionais. Fortaleza, Ceará, Brasil, 2001-2011.

\begin{tabular}{lccccccc}
\hline Variáveis & \multicolumn{7}{c}{ Secretarias Executivas Regionais } \\
& I & II & III & IV & V & VI & Centro \\
\hline Número de bairros & 14 & 19 & 15 & 21 & 18 & 29 & 3 \\
Proporção de bairros & 11,8 & 16,0 & 12,6 & 17,6 & 15,1 & 24,4 & 2,5 \\
Casos de AIDS em crianças & 20 & 5 & 20 & 7 & 17 & 16 & 1 \\
Número de crianças de 0 a 13 anos & 77.063 & 56.163 & 69.576 & 52.144 & 128.174 & 121.269 & 5.360 \\
Taxa de aids em crianças & 25,9 & 8,9 & 28,7 & 19,2 & 13,2 & 13,2 & 18,6 \\
Renda média & $1.003,6$ & $3.467,5$ & $1.129,6$ & $2.157,9$ & 749,7 & $1.313,2$ & $2.002,6$ \\
Proporção de analfabetos & 6,9 & 5,3 & 6,2 & 4,8 & 8,4 & 8,0 & 5,4 \\
Proporção de pessoas na faixa de pobreza & 5,0 & 3,2 & 4,6 & 3,2 & 6,5 & 5,5 & 3,3 \\
\hline
\end{tabular}

lação negativa e significativa entre a taxa de AIDS em crianças e os indicadores proporção de pobres $(r=-0,26 ; p=0,004)$ e proporção de analfabetos $(r=-0,27 ; p=0,02)$ (Tabela 2 ).

Regiões consideradas desfavoráveis, segundo indicadores utilizados neste trabalho, são aquelas com altas taxas de AIDS em crianças. As regiões da área de estudo que apresentaram maior proporção de analfabetos foram as que tiveram maior incidência de AIDS em crianças (Figuras 2 e 3).
Como resultado do modelo espacial multinomial, observaram-se aglomerados de casos de AIDS em crianças condicionados espacialmente aos fatores socioeconômicos, com riscos significativos em até duas vezes maiores nas áreas a noroeste e parte do sudoeste da cidade (Figura 4). 
Tabela 2

Correlação de Pearson da taxa de aids em criança com indicadores socioeconômicos e Índice de Moran da taxa de AIDS e dos indicadores socioeconômicos. Fortaleza, Ceará, Brasil, 2001-2011.

\begin{tabular}{|c|c|c|c|c|}
\hline \multirow[t]{2}{*}{ Indicadores sócio econômico } & \multicolumn{4}{|c|}{ Taxa de aids em criança } \\
\hline & r-Pearson & Valor de p & I Moran & Valor de $p$ \\
\hline Taxa de AIDS & & & -0.06 & 0.208 \\
\hline $\begin{array}{l}\text { Renda mensal dos } \\
\text { responsáveis }\end{array}$ & 0.15 & 0.1490 & 0.60 & 0.001 \\
\hline Proporção de pobres & -0.26 & 0.0040 & 0.48 & 0.001 \\
\hline Proporção de analfabetos & -0.27 & 0.0020 & 0.11 & 0.057 \\
\hline
\end{tabular}

Figura 2

Distribuição espacial por bairros da proporção de pessoas na faixa de pobreza e distribuição de renda média. Fortaleza, Ceará, Brasil, $2001-2011$.

\section{2a) Renda média}

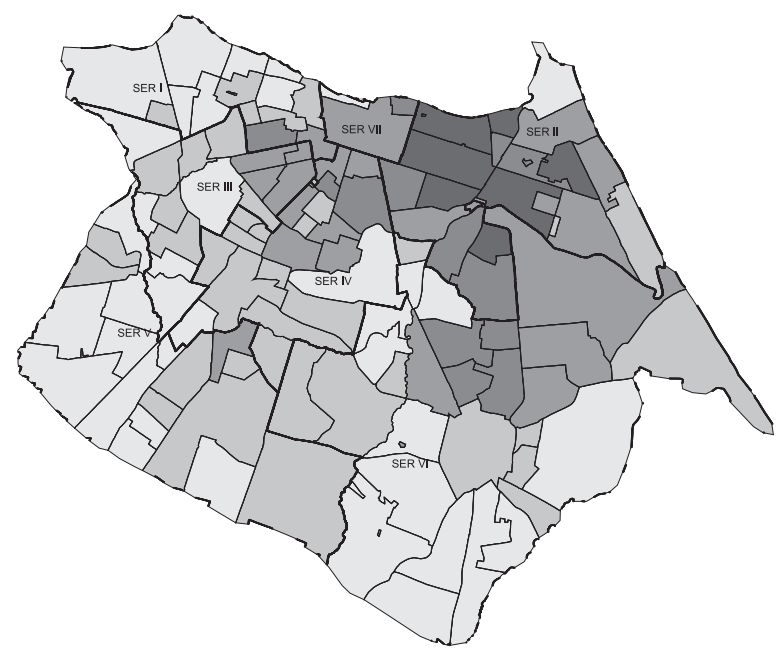

0 5 10 $20 \mathrm{~km}$

$R \$ 496,31-R \$ 974,82$

$R \$ 974,83-R \$ 1.557,25$

$R \$ 1.552,26-R \$ 2.621,70$

$R \$ 2.621,71-R \$ 4.493,48$

$R \$ 4.493,49-R \$ 7.697,39$ 2b) Faixa de pobres

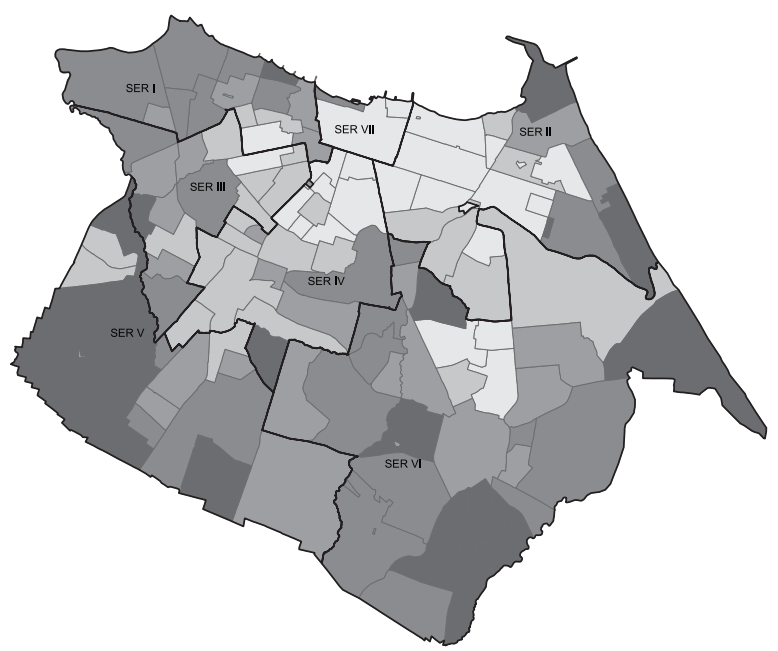

0

5

10 $20 \mathrm{~km}$
$0,57-1,91$
$1,92-3,82$
3,83-5,43
$5,44-7,43$
$7,44-9,42$ 
3a) AIDS em crianças

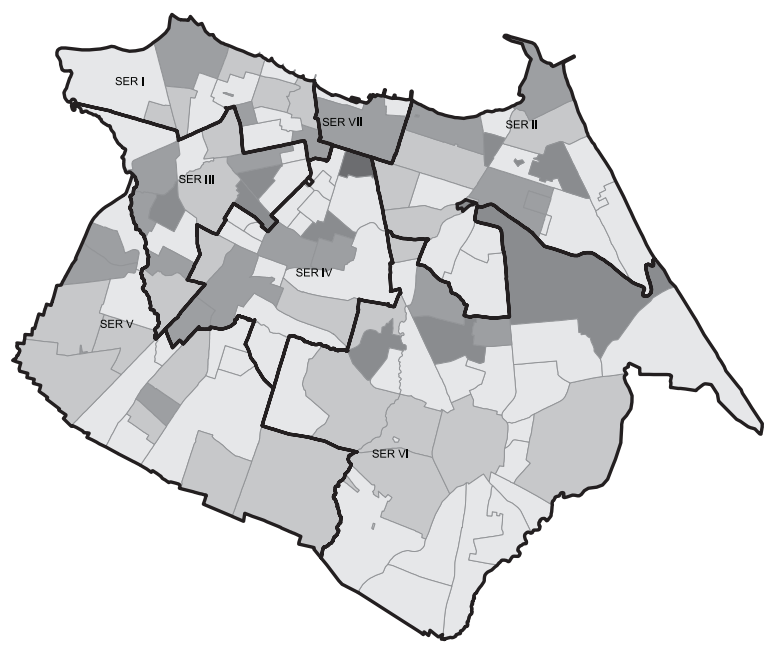

0

\section{$0,00-0,11$}

$0,12-0,43$

$0,44-0,86$

$0,87-1,65$

$1,66-2,88$ 3b) Analfabetos

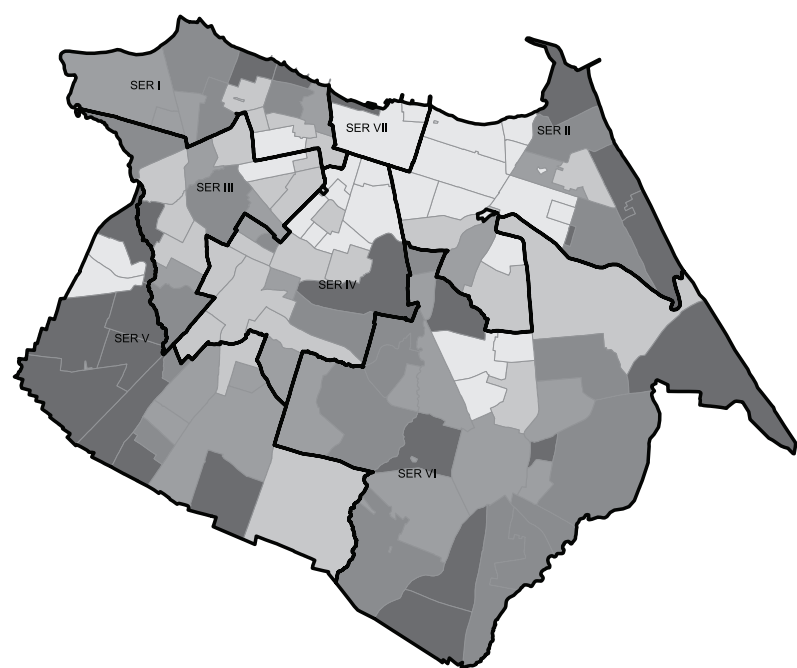

0

5

10

$20 \mathrm{~km}$

$1,26-3,29$

$3,30-5,37$

$5,38-7,30$

$7,31-9,87$

$9,88-14,37$

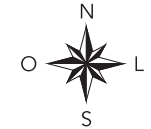

\section{Discussão}

A maior frequência de diagnóstico de AIDS em crianças ocorre aos dois anos de idade em função da indicação do Centers for Disease Control and Prevention (CDC) estabelecido até 2004, cuja idade de referência para a realização de testes para detecção de anticorpos anti-HIV era de 24 meses. O critério CDC é utilizado no Brasil desde 1987 e inclui sinais, sintomas e doenças, e exames laboratoriais. Entretanto, tais indicações são periodicamente atualizadas, visando a aumentar sua sensibilidade, adequando-se à realidade epidemiológica e aos avanços técnicos, científicos e organizacionais do Sistema Único de Saúde (SUS), com o propósito de garantir a notificação precoce dos casos e a redução do sub-registro ${ }^{12}$.

Ressalta-se que a adoção dos critérios seguidos no Brasil sofre influência das revisões e atualizações desenvolvidas por pesquisadores brasileiros e por estudos do CDC. Citam-se como exemplos: a identificação clínica de sinais, sintomas e doenças em detrimento à realização de testes diagnósticos que requerem técnicas mais sofisticadas; e os aspectos epidemiológicos do país, como a retirada da tuberculose da lista de doenças e agravos devido à sua alta prevalência e por possuir causas relativas às más condições de vida 12. Evidencia-se, assim, a importância do meio em que as pessoas vivem para a definição do perfil epidemiológico e tomada de decisões.

Analisando o ano de registro de notificações, no período estudado observa-se que o aumento da notificação de casos diagnosticados de AIDS ocorre após a instituição do Projeto Nascer Maternidades, em 2002, pelo Ministério da Saúde. A referida iniciativa teve como objetivo resgatar a oportunidade perdida de diagnóstico de HIV e 


\section{Figura 4}

Mapa temático de cluster de casos de AIDS em crianças. Fortaleza, Ceará, Brasil, 2001-2011.
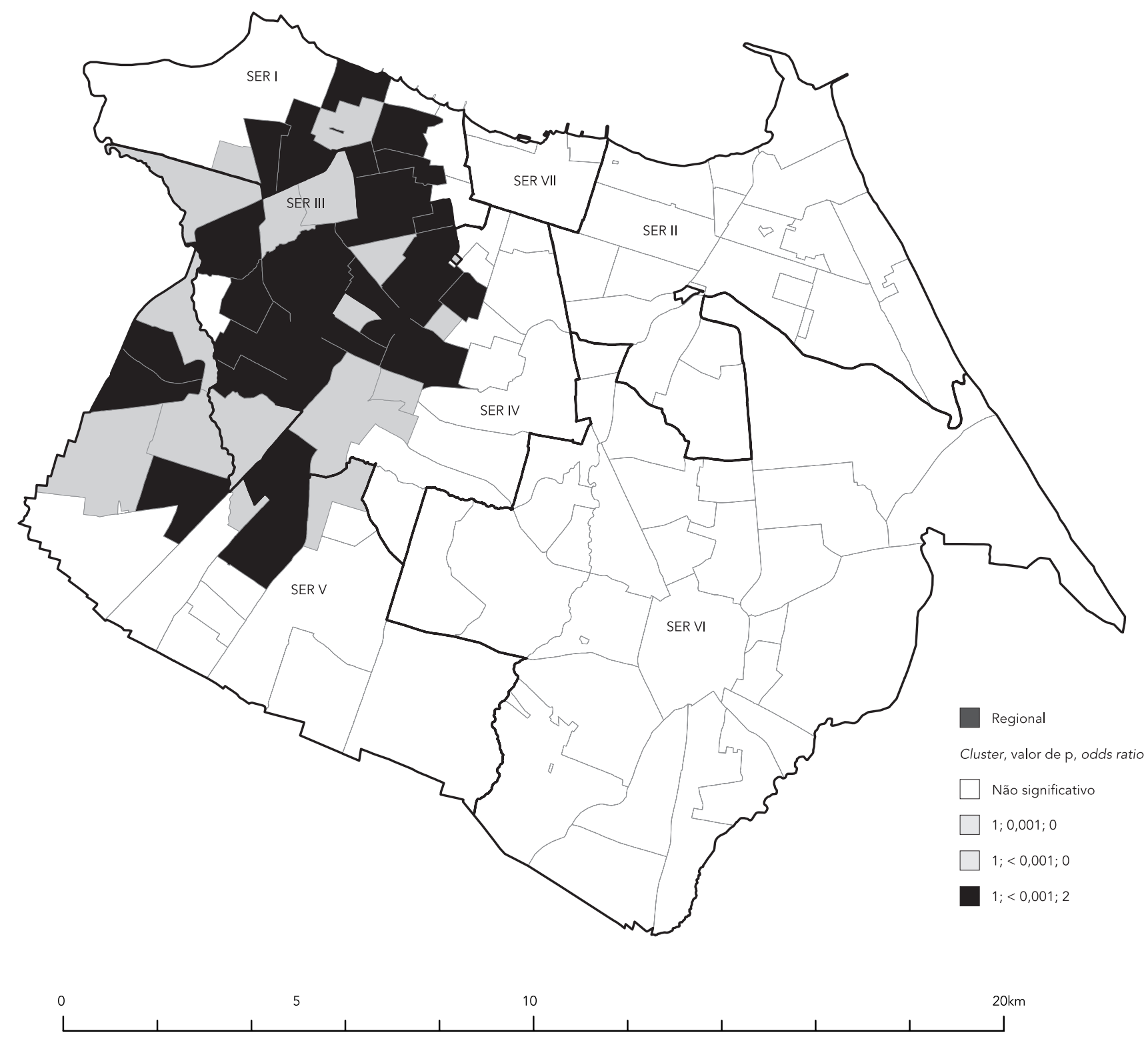

$\left.\circ\right|_{s} ^{N}$

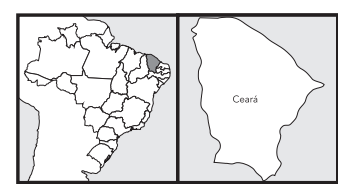


sífilis no pré-natal. Suas diretrizes determinam a realização de testes de todas as parturientes que não comprovem periparto, sorologias negativas para HIV e sífilis realizadas no pré-natal e a adoção de medidas profiláticas e/ou terapêuticas, se positividade dos exames 13. Outro aspecto é a possibilidade de melhora e mais agilidade da notificação, influenciada pelas mudanças de definição de casos, em 2004, o que pode ter contribuído para o aumento do quantitativo de casos observados a partir de 2002. Com essas intervenções de políticas públicas, contudo, esperava-se a diminuição do número de casos, entretanto este fato não foi observado.

No presente trabalho, evidencia-se diferença temporal entre o ano de diagnóstico e o ano de notificação. Os anos em que se verifica o maior número de casos diagnosticados não correspondem aos anos de maior quantitativo de casos notificados. Um estudo brasileiro baseado em duas pesquisas multicêntricas e cujo objeto foram os casos de crianças com AIDS no país aponta para o atraso na notificação; em $41,6 \%$ de crianças foram tardiamente notificados 14 . Uma investigação desenvolvida em Fortaleza indica diversos fatores que levam à subnotificação, como a não notificação dos casos pelo profissional médico, o atraso na investigação e a baixa qualidade da informação coletada que alimenta o SINAN. Essas situações podem favorecer a redução e a utilidade da notificação para acompanhamento da magnitude e tendência dessa epidemia 15 .

Na análise espacial, visualiza-se a relação geográfica dos casos de AIDS em crianças com os indicadores sociais: baixa renda média mensal, elevados índices de pessoas na faixa da pobreza e analfabetas. Os casos notificados em Fortaleza estão concentrados nos bairros da região noroeste e sudoeste da cidade, áreas onde são registradas condições de vida desfavoráveis à saúde.

A cidade de Fortaleza cresceu de forma ordenada em direção às zonas oeste e sul e em oposição ao litoral, surgindo bairros de maior renda média. Contudo, bairros que estão localizados nas extremidades da cidade apresentam maior incidência de pessoas em condição de extrema pobreza 16 . Tal fato pode ser explicado pela explosão demográfica ocorrida em meados da década de 1930, que acarretou um crescimento sem planejamento em direção às regiões periféricas, intensificando o surgimento de aglomerados subnormais desprovidos de infraestutura domiciliar e ordenamento urbanístico adequado 16,17. Os casos de AIDS estudados e sua distribuição de forma concentrada em determinadas regiões da cidade apontam para a necessidade urgente de intervenções que visem à redução de iniquidades sociais entre os bairros.
Dados semelhantes foram observados em outras regiões do Brasil, que apresentam maior concentração de renda, porém com regiões de pobreza, como as regiões Sul 4 e Sudeste 7. Autores identificaram maior risco para a transmissão vertical do HIV em áreas agrupadas ao longo de orla urbana pobre, onde há concentração de aglomerados subnormais e cujas condições de vida, como a baixa renda e viver em áreas socialmente desfavorecidas, são determinantes para as altas taxas de natalidade, que, por conseguinte, estão associadas à maior prevalência do HIV entre as mulheres grávidas ${ }^{4}$.

Um estudo local apontou a realidade vivida por mães de crianças com HIV, em que a renda familiar média foi de R\$ 446,32; as mulheres compartilhavam lares caracterizados por escassos recursos financeiros e $16,2 \%$ delas eram não escolarizadas 1 .

Os reduzidos recursos financeiros criam condições que alavancam os casos de AIDS e o HIV exacerba as múltiplas causas de interligação da pobreza, sendo as crianças severamente afetadas por este ciclo. Embora a educação, a melhoria da saúde e o acesso aos cuidados sejam importantes, o combate à pobreza deve ser um componente essencial da resposta a AIDS 18 .

$\mathrm{Na}$ realidade do enfrentamento à doença, uma das etapas é a identificação dos problemas que caracterizam a vulnerabilidade social, como a pobreza, a violência, o desemprego, pouco acesso às unidades de saúde e escolas e o conhecimento sobre o HIV/AIDS 19. Diante disso, para diminuir a vulnerabilidade social, são necessários programas de prevenção que atuem no interior do meio social.

Resultados de estudos como este devem embasar a tomada de decisões de políticas sociais. Contudo, corrobora-se a afirmação de que ainda há um longo e difícil caminho em busca da qualidade da assistência às pessoas com HIV/AIDS, em que superar iniquidades e desigualdades é um desafio 20. Mesmo anos após a Declaração de Compromissos para superar a AIDS, firmada nas Nações Unidas em 2001, a iniquidade social é citada como um desafio global e regional de característica complexa e que impulsiona essa epidemia 21.

No presente trabalho, outro fator que apresentou relação com os casos de AIDS foi a taxa de analfabetismo. Embora essa taxa na capital cearense tenha reduzido significativamente, ainda é considerada elevada e o número de analfabetos é composto não apenas pela população adulta, mas também por novas gerações não alfabetizadas, representadas principalmente por pessoas do sexo feminino 22. Em Fortaleza, um estudo que analisou as características das mães de crianças positi- 
vas para o HIV, constatou que metade delas tinha apenas o equivalente ao ensino fundamental 1.

A escolaridade exerce influência na adesão à terapia antirretroviral (TARV), que pode ter por consequência a transmissão do HIV da mãe para o filho, resultando nos casos de AIDS em crianças. Estudos realizados com gestantes positivas para o HIV internadas em hospitais evidenciaram a baixa escolaridade entre estas, o que foi associado às maiores taxa de infecção 23,24 . Em uma cidade do sul do Brasil, um estudo também demonstrou o predomínio de pessoas com baixa escolaridade vivendo com HIV/AIDS 25 . Na Nigéria, país responsável por $10 \%$ dos casos de infecção pelo HIV no mundo, um estudo mostrou que mais da metade das mulheres não sabiam qualquer método de prevenção da transmissão materno-infantil do HIV 26.

Evidencia-se, portanto, a necessidade de políticas governamentais e não governamentais que considerem a importância da avaliação de conhecimentos e atitudes sobre o HIV/AIDS e a transmissão vertical, como subsídios ao estabelecimento de programas de intervenção comunitária.

Os resultados deste trabalho corroboram com os de estudos anteriores que afirmam que a maioria dos fatores de risco ligados ao HIV/AIDS está associada às condições sociais e compartilham determinantes sociais com outros problemas importantes de saúde pública 27 .

A utilização do bairro como unidade de análise foi importante por ressaltar as diferenças sociais entre os bairros e evidenciar a ocorrência de casos de AIDS em crianças em setores nos quais se fazem necessárias intervenções políticas que busquem a melhoria das condições de vida da população.

A análise da distribuição espacial da pobreza é um importante fator a ser considerado em relação às políticas, que devem gerar ações capazes de dar respostas mais consistentes e com maior agilidade na geração de emprego e renda ${ }^{23}$. Os danos causados pela AIDS no Brasil revelam importantes diferenças regionais, mostrando a necessidade de melhorar a rede de serviços de saúde, com ênfase especial nas regiões menos desenvolvidas 28 .

No presente estudo, a análise espacial demonstrou que fatores socioeconômicos como pobreza e analfabetismo estão associados à ocorrência de casos de AIDS em crianças. Tal achado pode subsidiar o planejamento de ações preventivas e de promoção da saúde direcionadas para regiões da cidade identificadas como mais vulneráveis ao HIV.

$\mathrm{O}$ aconselhamento e testagem do HIV na atenção primária para gestantes na primeira consulta de pré-natal é uma das estratégias de prevenção e controle da transmissão do HIV/ AIDS. Entretanto, a ocorrência de AIDS em crianças pode ser considerada como consequência da transmissão materna, decorrente da ausência de diagnóstico durante o atendimento pré-natal 30. As consultas de pré-natal constituem uma importante ferramenta para o diagnóstico precoce e intervenções, possibilitando minimizar os riscos de infecção da criança. Contudo, os serviços de saúde, principalmente na atenção primária, ainda não abrangem a população em sua totalidade, principalmente em áreas onde as condições gerais de vida são desfavoráveis. Ademais, a ocorrência de AIDS em crianças pode ser considerada como negligência dos serviços de saúde no oferecimento de testagens e no seguimento em saúde de mulheres em risco de aquisição do vírus, repercutindo como consequência a transmissão do vírus durante a gestação e pós-parto.

Assim, além de políticas estritamente sociais voltadas para a redução de iniquidades, faz-se necessário ampliar e qualificar os serviços de saúde em suas atuações. Esses serviços devem orientar a população acerca de fatores que exercem influência sobre a condição biológica da comunidade atendida.

Destarte, os serviços de saúde devem ter sua prática balizada pela realidade na qual está inserida. Conhecer, além das características epidemiológicas, as características sociais e condições de vida, deve fazer parte do portfólio dos profissionais de saúde, principalmente no contexto da atenção primária. As condições de vida devem estar incluídas nos programas de ações e intervenções das equipes de saúde, bem como deve estar inserida em discussões populares e comunitárias, visto que esses profissionais são responsáveis ainda por estabelecer relação de empoderamento entre usuários e a rede de serviços prestados, incentivando os usuários mais participativos nas cobranças ao sistema de gestão por melhores condições de vida e saúde.

Apresentada a realidade da AIDS em crianças e sua relação com os aspectos socioeconômicos, acredita-se que o presente estudo possa ser considerado como uma ferramenta de ilustração da situação da AIDS em crianças e ser utilizado como suporte para decisões sociais e de saúde.

Apresenta-se, contudo, como limitação, o não preenchimento de algumas informações nas fichas de notificações de casos, como o endereço, ausente em algumas fichas, embora seja um dado essencial para a análise espacial e para o desenvolvimento de ações de prevenção e promoção da saúde nessa população. Cita-se ainda como limite as alterações de variáveis nas fichas de notificação, dificultando a contraposição de informações. A partir de 2007, houve mudança 
no instrumento, que foi dividido em ficha de notificação do HIV em gestantes e ficha de notificação de criança exposta. Importantes variáveis relacionadas à infecção pelo HIV e a atitudes da mãe antes e durante o pré-natal passaram a ser exclusivas da ficha de criança exposta. Contudo, essa ficha não consta como fonte de pesquisa nos bancos de dados dos serviços de saúde, o que leva à perda de importantes informações.

Sugere-se ainda estudos que tenham como objeto de estudo a consistência e a completude do preenchimento das fichas dos sistemas de notificação, com vista à busca constante da melhoria dos registros. O uso de banco de dados, uma vez satisfatório em seu preenchimento, reflete assertivamente a realidade dos serviços de saúde não apenas em um corte temporal, mas também, de forma prospectiva, permite analisar eventos em saúde e a eficácia de intervenções de políticas públicas. Outra sugestão refere-se a estudos que façam menção a intervenções de ações de promoção à saúde e prevenção de doenças e agravos. Estudos de intervenção, baseados em uma realidade diagnosticada e evidenciada, tornam-se mais específicos ao problema identificado e também se caracterizam como subsídios à tomada de decisões políticas e gerenciais para minimizar a ocorrência da infecção pelo HIV/AIDS e suas complicações no âmbito pessoal e social.
Tornam-se importantes também trabalhos que discutam temas que possam estabelecer novas relações com a ocorrência de casos de AIDS, fundamentando e favorecendo a tomada de decisões relativas à promoção da saúde e melhoria das condições de vida da população, sob novos prismas e novas possibilidades.

Em conclusão, na capital do Ceará, com a análise da distribuição espacial foi possível visualizar uma maior concentração de casos de AIDS em crianças em regiões com características sociais menos favorecidas, indicando a necessidade de implementação de programas direcionados ao atendimento de crianças, mães positivas para o HIV e às famílias, e o fortalecimento de redes sociais de apoio. Assim como é importante a efetivação de ações voltadas para a minimização da pobreza e melhoria da qualidade de vida da sociedade. Ainda, aponta-se para a necessidade de intervenções mais eficazes e efetivas no combate à transmissão e à transmissão vertical do HIV, considerando intervenções relativas ao contexto social em que vivem essas pessoas e famílias, principalmente em regiões socialmente desfavorecidas, pois são nestes locais onde tem sido constatado o maior número de casos e precisam, de modo urgente, de uma atenção voltada à sua qualidade de vida e saúde.

\section{Resumen}

El estudio tuvo como objetivo analizar la relación entre los casos de sida en niños y las características socioeconómicas de los barrios de Fortaleza, Ceará, Brasil. Se trata de un estudio ecológico y descriptivo, cuyos datos socioeconómicos se obtuvieron del Censo Demográfico de 2010, los datos de sida en niños - para el período 2001-20- se obtuvieron del Sistema de Notificación de Enfermedades. Se utilizó el análisis de correlación de Pearson y el índice de Moran para verificar la autocorrelación espacial. Se adoptó el modelo multinomial para identificar clusters. Hubo un 57,8\% de los niños varones. En un 8,8\%, el diagnóstico se produ- jo después de dos años de edad. Hubo una correlación positiva espacial entre los casos de sida en niños, en relación con los ingresos $(I=0,6 ; p=0,001)$ y la proporción de pobres $(I=0,5 ; p=0,001)$. Los vecindarios con esta caracterización están principalmente al noroeste y sudoeste de la ciudad. La correlación espacial de los casos de sida en niños con indicadores socioeconómicos destaca la necesidad de intervenciones dirigidas a mejorar las condiciones de vida y salud.

Sindrome de Inmunodeficiencia Adquirida; Niño; Factores Socioeconómicos 


\section{Colaboradores}

E. M. Lopes, R. L. F. Almeida e M. T. G. Galvão contribuíram na concepção e projeto ou análise e interpretação dos dados; redação do artigo e revisão crítica relevante do conteúdo intelectual; aprovação final da versão a ser publicada; e foram responsáveis por todos os aspectos do trabalho na garantia da exatidão e integridade de qualquer parte da obra. N. L. Pedrosa colaborou na concepção e projeto ou análise e interpretação dos dados; e na redação do artigo e revisão crítica relevante do conteúdo intelectual. E. R. Holanda e L. R. F. S. Kerr participou na concepção e projeto ou análise e interpretação dos dados; redação do artigo e revisão crítica relevante do conteúdo intelectual; e na aprovação final da versão a ser publicada.

\section{Referências}

1. Machado MMT, Galvão MTG, Lindsay AC, Cunha AJLA, Leite AJM, Leite RD, et al. Condições sociodemográficas de crianças de zero a dois anos filhas de mães com HIV/AIDS, Fortaleza, CE, Brasil. Rev Bras Saúde Matern Infant 2010; 10:377-82.

2. Schaurich D, Medeiros HMF, Motta MGC. Vulnerabilidades no viver de crianças com AIDS. Rev Enferm UERJ 2007; 15:284-90.

3. Ministério da Saúde. Boletim Epidemiológico AIDS e DST 2012; Ano I. oo 1.

4. Barcellos C, Acosta LMW, Lisboa E, Bastos FI. Surveillance of mother-to-child HIV transmission: socioeconomic and health care coverage indicators. Rev Saúde Pública 2009; 43:1006-14.

5. Stephan C, Henn CA, Donalisio MR. Expressão geográfica da epidemia de AIDS em Campinas, São Paulo, de 1980 a 2005. Rev Saúde Pública 2010; 44:812-9.
6. Joint United Nations Program on HIV/AIDS. Investing for results. Results for people. A peoplecentred investment tool towards ending AIDS. Geneva: World Health Organization; 2012.

7. Bailey TC, Gatrell AC. Spatial data analysis. $1^{\text {st }}$ Ed. Essex: Longman Scientific \& Technical; 1995.

8. Vieira ACBC, Miranda AE, Vargas PRM, Maciel ELN. Prevalência de HIV em gestantes e transmissão vertical segundo perfil socioeconômico, Vitória, ES. Rev Saúde Pública 2011; 45:644-51.

9. Núcleo de Epidemiologia, Coordenadoria de Promoção e Proteção à Saúde, Secretaria da Saúde. Informe epidemiológico. AIDS 2011. Fortaleza: Secretaria da Saúde; 2011.

10. Romero DE, Cunha AB. Avaliação da qualidade das variáveis epidemiológicas e demográficas do Sistema de Informações sobre Nascidos Vivos, 2002. Cad Saúde Pública 2007; 23:701-14. 
11. Hosmer DW, Lemeshow S. Model building strategies and methods for logistic regression. In: Lemeshow S, Sturdivant RX, Hosmer DW, editors. Applied logistic regression. Hoboken: John Wiley \& Sons; 2013. p. 83-133.

12. Secretaria de Vigilância em Saúde, Ministério da Saúde. Critérios de definição de casos de AIDS em adultos e crianças. Brasília: Ministério da Saúde; 2004.

13. Ministério da Saúde. Portaria no 2.104 de 19 de novembro de 2002. Institui no âmbito do Sistema Único de Saúde - SUS o Projeto Nascer-Maternidades. Diário Oficial da União 2002; 20 nov.

14. Ramos Jr. AN, Matida LH, Alencar CH, Hearst N, Heukelbach J. Late-stage diagnosis of HIV infection in Brazilian children: evidence from two national cohort studies. Cad Saúde Pública 2013; 29(7):1291-300.

15. Gonçalves VE, Kerr LRFS, Mota RMS, Mota JMA. Estimativa de subnotificação de casos de AIDS em uma capital do Nordeste. Rev Bras Epidemiol 2008; 11:356-6.

16. Instituto de Pesquisa e Estratégia Econômica do Ceará. Perfil municipal de Fortaleza. Tema VIII: O mapa da extrema pobreza. Edição Especial. Fortaleza: Instituto de Pesquisa e Estratégia Econômica do Ceará; 2012. (Informe, 43).

17. Bruno A, Farias JA. Fortaleza: uma breve história. Fortaleza: Instituto de Pesquisa e Estratégia Econômica do Ceára; 2011.

18. Marais BJ, Esser M, Godwin S, Rabie H, Cotton MF. Poverty and human immunodeficiency virus in children: a view from the Western Cape, South Africa. Ann NY Acad Sci 2008; 1136:21-7.

19. Programa Nacional de DST e AIDS, Secretaria de Vigilância em Saúde, Ministério da Saúde. Manual de prevenção das DST/HIV/AIDS em comunidades populares. Brasília: Ministério da Saúde; 2008.

20. Villarinho MV, Padilha MI, Berardinelli LMM, Borenstein MSB, Meirelles BHS, Andrade SR. Políticas públicas de saúde face a epidemia da AIDS e a assistência às pessoas com a doença. Rev Bras Enferm 2013; 66:271-7.

21. Departamento de DST, AIDS e Hepatites Virais, Secretaria de Vigilância em Saúde, Ministério da Saúde. Relatório de Progresso da Resposta Brasileira ao HIV/AIDS (2010-2011). Brasília: Ministério da Saúde; 2012.
22. Instituto de Pesquisa e Estratégia Econômica do Ceará. Perfil socioeconômico de Fortaleza. Fortaleza: Instituto de Pesquisa e Estratégia Econômica do Ceará; 2012

23. Konopka CK, Beck ST, Wiggers D, Silva AK, Diehl FP, Santos FG. Perfil clínico e epidemiológico de gestantes infectadas pelo HIV em um serviço do sul do Brasil. Rev Bras Ginecol Obstet 2010; 32:18490.

24. Sanz SM, Guinsburg R. Prevalência da soropositividade para sífilis e HIV em gestantes de um hospital de referência materno infantil do Estado do Pará. Rev Pará Med 2008; 22(3). http://files.bvs. br/upload/S/0101-5907/2008/v22n3/a2277.pdf (acessado em 13/Dez/2013).

25. Schuelter-Trevisol F, Pucci P, Justino AZ, Pucci N, Silva ACB. Perfil epidemiológico dos pacientes com HIV atendidos no sul do Estado de Santa Catarina, Brasil, em 2010. Epidemiol Serv Saúde 2013; 22:87-94.

26. Adeleke SI, Mukhtar-Yola M, Gwarzo GD. Awareness and knowledge of mother-to-child transmission of HIV among mothers attending the pediatric HIV clinic, Kano, Nigeria. Ann Afr Med 2009; 8:210-4.

27. Organização Mundial da Saúde. Diminuindo as diferenças: a prática das políticas sobre determinantes sociais da saúde. Geneva: Organização Mundial da Saúde; 2011. (Documento de Discussão).

28. Ramos Junior AN, Matida LH, Hearst N, Heukelbach J. AIDS morbidity and mortality in Brazilian children before and after highly active antirretroviral treatment implementation: an assessment of regional trends. Pediatr Infect Dis J 2011; 30:773-7.

29. Teixeira TRA, Gracie R, Malta MS, Bastos FI. Social geography of AIDS in Brazil: identifying patterns of regional inequalities. Cad Saúde Pública 2014; 30:259-71.

30. Araújo MAL, Vieira NFC, Araújo CLF. Aconselhamento coletivo pré-teste anti-HIV no pré-natal: uma análise sob a ótica dos profissionais de saúde. Rev Baiana Saúde Pública 2009; 33:122-35.

Recebido em 16/Jul/2014

Versão final reapresentada em 14/Jan/2015

Aprovado em 05/Mar/2015 